\title{
CLEAR VISIBILITY AND SETS WHICH ARE ALMOST STARSHAPED
}

\author{
MARILYN BREEN
}

\begin{abstract}
Let $S$ be a nonempty compact connected set in $R^{2}$. Assume that every two points of local nonconvexity of $S$ are clearly visible from a common point of $S$. Then $S$ is "almost" starshaped. In fact, for any point $y$ in $R^{2}$ there is a line $L$ through $y$ such that $L \cap S$ is convex and every point of $S$ sees via $S$ a point of $L$. If "clearly visible" is replaced by the weaker term "visible", then the result fails.
\end{abstract}

1. Introduction. We begin with some preliminary definitions. Let $S$ be a subset of the plane. A point $s$ in $S$ is said to be a point of local convexity of $S$ if and only if there is some neighborhood $N$ of $s$ such that $N \cap S$ is convex. If $S$ fails to be locally convex at point $q$ in $S$, then $q$ is called a point of local nonconvexity (lnc point) of $S$. For points $x$ and $y$ in $S$, we say $x$ sees $y$ via $S$ ( $x$ is visible from $y$ via $S$ ) if and only if the corresponding segment $[x, y]$ lies in $S$. Point $x$ is clearly visible from $y$ via $S$ if and only if there is some neighborhood $N$ of $x$ such that $y$ sees each point of $S \cap N$ via $S$. Set $S$ is an $L_{2}$ set if and only if every two points of $S$ are visible via $S$ from a common point. Finally, $S$ is starshaped if and only if there is some point $p$ in $S$ such that $p$ sees each point of $S$ via $S$, and the set of all such points $p$ is called the (convex) kernel of $S$, denoted ker $S$.

A well-known theorem of Krasnosel'skir [4] states that if $S$ is a nonempty compact set in $R^{2}$, then $S$ is starshaped if and only if every 3 points of $S$ are visible via $S$ from a common point. If the number 3 is reduced to 2 , then the result fails, for a compact $L_{2}$ set need not be even a finite union of starshaped sets [2, Example 1]. However, this leads to a related question examined in [2]: When will a closed planar set be "almost" starshaped? That is, when will there exist a convex subset $C$ of $S$ such that every point of $S$ sees via $S$ some point of $C$ ?

In several studies of starshaped sets, the notion of clearly visible has been a useful tool. Here we use this concept to obtain the following result: Let $S$ be a nonempty compact connected set in $R^{2}$. Assume that every 2 points of local nonconvexity of $S$ are clearly visible from a common point of $S$. Then $S$ is almost starshaped. In fact, for any point $y$ in $R^{2}$ there exists a line $L$ through $y$ such that $L \cap S$ is convex and every point of $S$ sees via $S$ a point of $L$. If "clearly visible" is replaced by the weaker term "visible", then the result fails.

The following familiar terminology will be used throughout the paper: conv $S$ and $\operatorname{cl} S$ will denote the convex hull and closure, respectively, for set $S$, and lnc $S$ will be the set of points of local nonconvexity of $S$. For distinct points $x$ and $y, L(x, y)$ will be the line determined by $x$ and $y$, and $R(x, y)$ will be the ray from

Received by the editors August 5, 1983.

1980 Mathematics Subject Classification. Primary 52A30, 52A35.

(c) 1984 American Mathematical Society $0002-9939 / 84 \$ 1.00+\$ .25$ per page 
$x$ emanating through $y$. Finally, cone $(x, A)$ will represent $\bigcup\{R(x, a): a \in A, a \neq x\}$. The reader is referred to Valentine [7] and Lay [5] for a discussion of these concepts.

2. The results. We will be concerned with the proof of the following theorem.

THEOREM 1. Let $S$ be a nonempty compact connected set in $R^{2}$. Assume that every 2 points of local nonconvexity of $S$ are clearly visible from a common point. Then $S$ is "almost" starshaped. In fact, for any point $y$ in $R^{2}$ there is a line $L$ through y such that $L \cap S$ is convex and every point of $S$ sees via $S$ some point of $L$.

Proof. If $S$ is convex, the result is immediate. Thus we may assume that $S$ is not convex and hence has lnc points by a theorem of Tietze [6]. For each lnc point $q$ of $S$, define the set $A_{q}=\{x: q$ is clearly visible from $x$ via $S\}$. Using our hypothesis, every 2 of the compact convex sets in $A \equiv\left\{\operatorname{conv} \operatorname{cl} A_{q}: q\right.$ in $\left.\operatorname{lnc} S\right\}$ intersect. Therefore, by a result of Horn [3, Theorem 4], for any point $y$ in $R^{2}$ there is a line $L$ through $y$ which intersects every member of $A$. We will show that $L$ satisfies the theorem.

The proof will be accomplished by a sequence of assertions.

ASSERTION 1. $S \cap L$ is convex: Suppose on the contrary that there exist points $a, b \in S \cap L$ with $(a, b) \cap S=\emptyset$, to obtain a contradiction. By [8, Lemma 1], each point of $S$ sees via $S$ some lnc point, so $a$ sees an lnc point, as does $b$. Moreover, by hypothesis, every 2 lnc points are visible from a common point of $S$. Hence there is a polygonal path $\lambda$ in $S$ from $a$ to $b$, where $\lambda$ has length at most 4 and $\lambda$ has no multiple points. Choose $a^{\prime}, b^{\prime} \in \lambda \cap L$ such that $a^{\prime} \leq a<b \leq b^{\prime}$ and such that there are no points of $L$ on the subpath $\lambda^{\prime}$ of $\lambda$ joining $a^{\prime}$ to $b^{\prime}$ (except for $a^{\prime}$ and $b^{\prime}$, of course).

Consider the closed simply connected subset $T$ of $R^{2}$ bounded by $\lambda^{\prime} \cup\left[a^{\prime}, b^{\prime}\right]$. Let $K$ be the component of $T \sim S$ which contains $(a, b)$. Let $L^{\prime}$ be a line parallel to $L$ and distinct from $L$ supporting conv $\mathrm{cl} K$, and let $q$ be an extreme point of conv cl $K$ on $L^{\prime}$. Using an argument similar to the one in [1, Lemma 3, Case 1], it is not hard to show that $q$ is an lnc point of $S$ and $q$ is clearly visible via $S$ only from points in the closed halfspace $\mathrm{cl} L_{1}^{\prime}$ determined by $L^{\prime}$ and disjoint from $L$. However, this implies that conv $\operatorname{cl} A_{q} \subseteq \operatorname{cl} L_{1}^{\prime} \subseteq \sim L$, impossible since (convcl $\left.A_{q}\right) \cap L \neq \emptyset$. We have a contradiction, and $L \cap S$ must be convex.

ASSERTION 2. For $q \in \operatorname{lnc} S, q$ sees via $S$ some point of $L \cap \operatorname{cone}\left(q, \operatorname{cl} A_{q}\right)$ : By our choice of $L$, it is easy to see that $\operatorname{cl} A_{q}$ cannot lie in either of the open halfplanes $L_{1}$ or $L_{2}$ determined by $L$. Hence we may select points $p \in \operatorname{cl} A_{q} \cap \operatorname{cl} L_{1}$ and $r \in \operatorname{cl} A_{q} \cap \operatorname{cl} L_{2}$. Without loss of generality, assume that $q \in \operatorname{cl} L_{1}$. Then $[q, r]$ meets $L$ at some point $s$, and $s$ satisfies the assertion.

ASSERTION 3. Set $S$ is simply connected: Suppose on the contrary that $\sim S$ contains a bounded component $B$. Let $M$ and $N$ be distinct parallel lines supporting $\operatorname{conv} \operatorname{cl} B$, and let $c$ and $d$ be extreme points of $\operatorname{conv} \operatorname{cl} B$ in $M$ and in $N$, respectively. Using an argument from [1, Lemma 3, Case 1], $c$ and $d$ are lnc points of $S$. Moreover, if the corresponding halfplanes are labeled so that $\operatorname{cl} M_{1} \cap \mathrm{cl} N_{2}=\emptyset$, then $A_{c} \subseteq \mathrm{cl} M_{1}, A_{d} \subseteq \mathrm{cl} N_{2}$, and $c$ and $d$ cannot be clearly visible from a common point of $S$, contradicting our hypothesis.

ASSERTION 4. For $x$ in $S, x$ sees via $S$ some point of line $L$ : If $x$ is an lnc point, the argument is finished by Assertion 2 above, so assume that $x$ is not an 


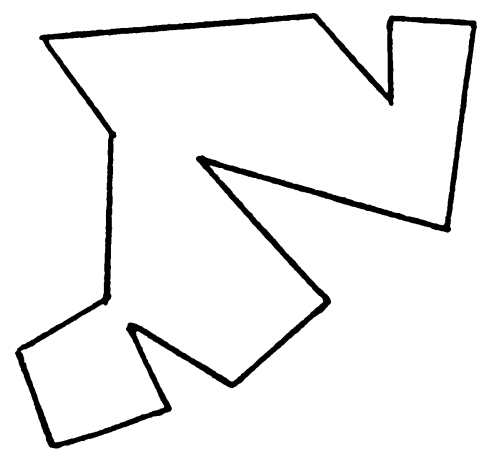

FIGURE 1

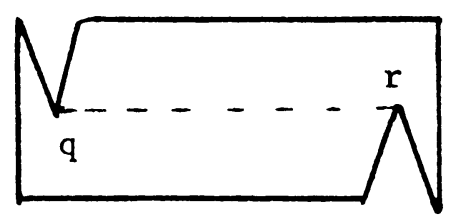

FIGURE 2

lnc point. By [8, Lemma 1], $x$ sees via $S$ some lnc point $q$. Moreover, by Assertion $2, q$ sees via $S$ some point $p \in L \cap \operatorname{cone}\left(q, \operatorname{cl} A_{q}\right)$. Now if $[x, q]$ meets $L$ or if $x, q, p$ are collinear, we are finished. Otherwise, choose point $w$ on $[q, p]$ closest to $p$ such that $\operatorname{conv}\{x, q, w\} \subseteq S$. If $w=p$, again we are through, so assume $w \neq p$. Then by an argument involving [8, Corollary 1], $[x, w]$ contains an lnc point $q_{2}$ of $\operatorname{conv}\{x, q, p\} \cap S$. Clearly $q_{2} \in \operatorname{lnc} S$ and since $x \notin \operatorname{lnc} S, q_{2} \neq x$. Moreover, it is not hard to see that $A_{q_{2}}$ lies in the closed halfplane cl $M_{2}$ determined by $M \equiv L(x, w)$ and disjoint from $p$. Again using Assertion 2, $q_{2}$ sees via $S$ some point $p_{2}$ of $L \cap$ cone $\left(q_{2}, \operatorname{cl} A_{q_{2}}\right)$, and since $A_{q_{2}} \subseteq \operatorname{cl} M_{2}, p_{2} \in \operatorname{cl} M_{2}$. Observe that $L$ and $L(x, w)$ cannot be parallel, and let $r$ denote their point of intersection. Then $p_{2} \leq r<p$ on $L$. Since $L \cap S$ is convex, $\left[p_{2}, p\right] \subseteq S$. Hence

$$
[x, q] \cup[q, w] \cup\left[w, q_{2}\right] \cup\left[q_{2}, p_{2}\right] \cup\left[p_{2}, p\right] \cup[p, w] \cup[w, x] \subseteq S .
$$

Using the fact that $S$ is simply connected, it is easy to see that $[x, r] \subseteq S$, and the assertion is established.

This completes the proof of the theorem.

It is interesting to note that the result in Theorem 1 fails if "clearly visible" is replaced by the weaker term "visible", as the following example illustrates.

EXAMPLE 1. Let $S$ be the set in Figure 1. Every 2 (in fact, all) lnc points of $S$ are visible from a common point, yet $S$ is not almost starshaped.

Finally, the condition in Theorem 1 is sufficient but not necessary for a set to be almost starshaped, as Example 2 reveals.

EXAMPLE 2. Let $S$ be the set in Figure 2. The 2 lnc points $q$ and $r$ of $S$ are clearly visible from no common point, yet every point of $S$ sees via $S$ some point of segment $[q, r]$. 


\section{REFERENCES}

1. Marilyn Breen, A Krasnosel' skii-type theorem for points of local nonconvexity, Proc. Amer. Math. Soc. 85 (1982), 261-266.

2. __, $L_{2}$ sets which are almost starshaped, Geom. Dedicata 6 (1977), 485-494.

3. Alfred Horn, Some generalizations of Helly's theorem on convex sets, Bull. Amer. Math. Soc. 55 (1949), 923-929.

4. M. A. Krasnosel'skii, Sur un critère pour qu'un domain soit étoilé, Math. USSR-Sb. 19 (61) (1946), 309-310.

5. Steven R. Lay, Convex sets and their applications, Wiley, New York, 1982.

6. H. Tietze, Über Konvexheit im kleinen und im grossen und über gewisse den Punkten einer Menge zugeodnete Dimensionzahlen, Math. Z. 29 (1928), 697-707.

7. F. A. Valentine, Convex sets, McGraw-Hill, New York, 1964.

8. __ Local convexity and $L_{n}$ sets, Proc. Amer. Math. Soc. 16 (1965), 1305-1310.

DePaRtMENT OF MATHEMATICS, UNIVERSity OF OKLAHOMA, NORMAN, OKLAHOMA 73019 